Journal of Statistical Mechanics:

Theory and Experiment

PAPER: QUANTUM STATISTICAL PHYSICS, CONDENSED MATTER, INTEGRABLE SYSTEMS

$n$-cluster models in a transverse magnetic field

To cite this article: G Zonzo and S M Giampaolo J. Stat. Mech. (2018) 063103

View the article online for updates and enhancements. 


\title{
n-cluster models in a transverse magnetic field
}

\section{G Zonzo ${ }^{1}$ and S M Giampaolo S $^{2,3}$}

${ }^{1}$ Dipartimento di Fisica, Università degli Studi di Salerno, Via Giovanni Paolo II 132, I-84084 Fisciano (SA), Italy

2 Division of Theoretical Physics, Ruđer Bošković Institute, Bijenćka cesta 54, 10000 Zagreb, Croatia

${ }^{3}$ International Institute of Physics, UFRN, Av. Odilon Gomes de Lima 1722, 59078-400 Natal, Brazil

E-mail: smgiampaolo72@gmail.com

Received 8 February 2018

Accepted for publication 9 May 2018

Published 11 June 2018

Online at stacks.iop.org/JSTAT/2018/063103

https://doi.org/10.1088/1742-5468/aac443

\begin{abstract}
We analyze a family of 1D fully analytically solvable models in which a many-body cluster interaction, acting simulatenously on $n+2$ spins, competes with a uniform transverse external field. These models can be solved analytically using the Jordan-Wigner transformations and we prove that they present a very rich phase diagram with both nematic and symmetry protected topological ordered phases. From the point of view of the entanglement, these models show a non vanishing bipartite entanglement between the spins at the end points of the cluster term. At the same time, regardless to the system parameters, it is possible to prove analytically that there in no genuine multipartite entanglement among the spins of a subset made by $m \leqslant n+2$. Numerical simulations suggest that this absence extends also to larger subsets. Due to their integrability and to the peculiar entanglement properties, the $n$-cluster models in a transverse magnetic field may serve as a prototype for studying non trivial order and can be of extreme relevance for applications of quantum information tasks.
\end{abstract}

Keywords: quantum phase transitions, spin chains, ladders and planes, entanglement in extended quantum systems, correlation functions 


\section{Contents}

1. Introduction 2

2. Solution of the family of models 4

2.1. Ground state and energies .................................................................... 5

2.2. Fermionic correlations functions....................................................... 7

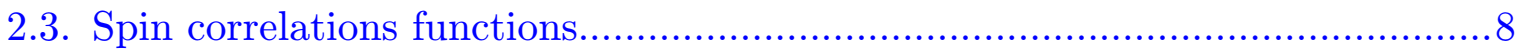

3. Phase diagrams and order parameters 9

4. The entanglement properties $\quad 12$

4.1. Entanglement between two spins ........................................................ 13

4.2. Genuine multipartite entanglement ....................................................... 14

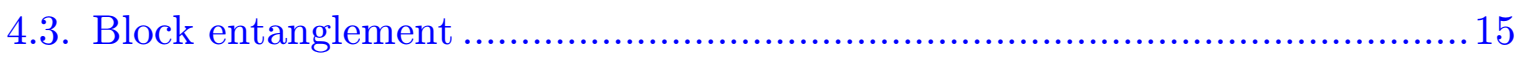

5. Conclusions $\quad 17$

Acknowledgments ............................................................. 18

$\begin{array}{ll}\text { References } & 18\end{array}$

\section{Introduction}

The scientific community has now widely accepted the fact that the analysis of the entanglement properties of the ground states can provide the most fundamental characterization of a quantum many-body system. Indeed the study of the entanglement can characterize the quantum critical point [1-3] exactly as the Ginzburg-Landau approach directly borrowed from the classical statistical mechanics [4, 5]. But it can also unveil several other aspects that cannot be detected by the standard analysis and that have shed a new light on quantum many-body systems. To give an example it was discovered that quantum non critical systems obey to the so called Area-Law, i.e. the fact that the entanglement entropy scales with the area of the subregion and not with its volume [6-12]. Moreover non trivial sub-leading terms of the von Neumann entropy can signal the presence of topological ordered phase [13-15]. Also the total absence of the entanglement in the so called factorization point [16-18] allow to characterize the magnetically ordered phases and make possible to predict phase transitions by analyzing the dynamics of a finite size system [19].

However, although now the entanglement is recognized as one of the fundamental concepts of the quantum physics, its evaluation is still an open problem. With the exception of few simple cases there is no necessary and sufficient general way to measure the entanglement. This is especially true in the case of genuine multipartite entanglement. Consequently the analysis of entanglement properties of the ground state of a many-body system was focused mainly on the bipartite entanglement. But, in recent years, this situation is slowly changing. Although the difficulties in quantifying the 
multipartite entanglement are still present, several studies have highlighted its presence and role of in the $X Y$-model [20,21]. Moreover it has also been shown that models with cluster interactions can be characterized simultaneously by the total absence of bipartite entanglement between two spins and the presence of genuine multipartite entanglement between three or more elements of the system [22, 23]. At first sight, this result would seem to be connected to the fact that an interaction that acts on several elements of the system simultaneously, as the cluster one, should naturally tend to generate multipartite entanglement.

This interpretation, as we will show in the present work, is not correct. Indeed the cluster interaction, interplaying with an external field, allow to obtain a system in which we have both a non vanishing bipartite entanglement and a zero multipartite entanglement at the same time. Strictly speaking, to provide the proof of the complete absence of any kind of genuine multipartite entanglement we would have to analyze any possible subset of spins of the system for all possible values of the parameters. Such kind of analysis is impossible to do not only because of the numberless different subsets to analyze but also because of the lack of a general closed formula for the genuine multipartite entanglement. Therefore in our analysis we: (a) use the peculiar properties of the family of models under study to prove, analytically, that there is no genuine multipartite entanglement in any subset made by a number of spin less or equal to the dimension of the cluster interaction term; (b) make a numerical analysis, based on a sufficient but not necessary criterion [24, 25], to support the idea that, also subsets of spins larger than the cluster term do not show any genuine multipartite entanglement.

But the peculiar entanglement properties is not the only reason to study the $n$-cluster models in external fields. In fact, the models also show two novel ordered phases of matter that fall outside the Ginzburg-Landau scheme, i.e. the nematic and the symmetry protected topological phases. Nematic phases [26, 27] occur in a system in which we have a ground state that: (a) breaks at least one symmetry of the Hamiltonian; (b) is characterized by an order parameter with a support defined on a finite number of spins $\ell$ with $\ell>1$. In some senses, the nematic order can be seen as a generalization of the magnetic one to the case in which the order parameter is not strictly local. On the other hand the symmetry protected topological ordered phases are characterized by string order parameters, i.e. non vanishing expectation value of operators which support extends on the whole system [28, 29]. Topological ordered phases are associated to the robustness of ground state degeneracies [13], show quantized non-Abelian geometric phases [14] and play a fundamental role both in the spin liquids [30,31] and in non-Abelian fractional Hall systems [32]. Moreover they will play a key role in the development of fault-tolerant quantum computers [33]. On the other hand, nematic order is usually found in materials commercially used in liquid crystal technology [34]. Therefore it is not surprisingly that the number of papers devoted to their analysis is continuously increasing. To limit ourselves to the 1D spin systems, it is known that the frustrated 1D spin-1/2 chain in an external magnetic field shows a nematic ordered phase [35, 36], the $1 \mathrm{D}$ cluster Ising model exhibits a symmetry-protected topological ordered phase $[22,37,38]$ and the $n$-cluster Ising models, that can obtained using Floquet interactions in atomic systems [39], show both nematic and topological orders, depending on $n$ [23]. 
The paper is organized as follow. In section 2 we define the family of models that we are considering and show how it can be analytically solved using the JordanWigner transformations [40]. We will prove that all the models of the family hold a quantum critical point when the relative weights of the external field and of the cluster interaction are the same. The quantum critical point separates two phases: a paramagnetic phase, in which the external field dominates on the cluster interaction, and an ordered cluster phase when the many-body interaction is more relevant than the field. The ordered cluster phase can be a nematic or a topological phase depending on the number of spins involved in the many-body interaction. In both cases we determine and analyze the order parameter (see section 3). In section 4, we start to analyze the entanglement properties starting from the correlation functions that we have derived in section 2.3. At first, we focus on the concurrence that measures the entanglement between two spins [41, 42]. We will show that, in contrast with the $n$-cluster Ising model [23], for any value of $n$, there is a region of the parameter $\phi$ for which the entanglement between the two spins at the end points of a cluster term differs from zero. Then we move our analysis on the genuine multipartite entanglement. Making use of the particular symmetries of the system we will prove analytically that for a block made by $m \leqslant n+2$ spins there is no genuine multipartite entanglement. For larger subsets, because of the lack of a closed formula valid for all states, it is impossible to prove the absence of the multipartite entanglement. We made several numerical evaluations using the sufficient but not necessary criterion developed in [24, 25]. This criterion, if verified, proves the presence of the genuine multipartite entanglement but, if not, does not give any conclusive response. In all our numerical simulation the criterion is never verified driving us to think that the genuine multipartite entanglement vanishes also in subsets of spins larger than the cluster size. Before to conclude we move to analyze the block entanglement, at the quantum phase transition $\phi=\phi_{\mathrm{c}} \equiv \pi / 4$ and by using the conformal field theory [10] we evaluate the central charge of the models, that turns out to be dependent on $n$, proving that the models of the family fall into different classes of symmetry. In section 5, we draw our conclusions.

\section{Solution of the family of models}

We focused our analysis on a family of $1 \mathrm{D}$ spin- $1 / 2$ models characterized by the interplay between a cluster interaction and an external field. The Hamiltonian of such family of models can be written as

$$
H_{\phi}^{(n)}=-J \cos (\phi) \sum_{j} \sigma_{j}^{x} O_{j, n}^{z} \sigma_{j+n+1}^{x}+J \sin (\phi) \sum_{j} \sigma_{j}^{z} .
$$

The parameter $\phi$ controls the relative weight of the two terms, $J$ has the dimension of an energy, $\sigma_{i}^{\alpha}$ (with $\alpha=x, y, z$ ) are the Pauli operators and $O_{j, n}^{z}$ stands for

$$
O_{j, n}^{z}=\bigotimes_{k=1}^{n} \sigma_{j+k}^{z} .
$$


The Hamiltonian in equation (1) can be solved analytically by using the JordanWigner transformation [40]. Such transformation maps spin models into a system made by non-interacting spinless fermions [23, 37, 43-45]. Applying the Jordan-Wigner transformation to equation (1), after some algebras, we obtain

$$
\begin{aligned}
H_{\phi}^{(n)}= & J \cos (\phi) \sum_{j}\left(c_{j}^{\dagger} c_{j+n+1}+c_{j}^{\dagger} c_{j+n+1}^{\dagger}+\text { h.c. }\right) \\
& -J \sin (\phi) \sum_{j}\left(2 c_{j}^{\dagger} c_{j}-1\right)
\end{aligned}
$$

where $c_{i}\left(c_{i}^{\dagger}\right)$ it the operator that annihilate (create) a spinless fermions on the site $i$.

At first sight, the fermionic Hamiltonian in equation (3) is very close to the one obtained applying the Jordan-Wigner transformation to the quantum Ising model in the external field [44]. The main difference is that, while in the second case, each interaction term involves two fermionic sites at distance 1, in the present case the interactions is realized between sites at distance $n+1$. One can be tempted to re-arrange the different terms in such a way that the model under analysis can be seen as a set of $n+1$ independent fermionic systems with interaction between nearest sites. But this re-arrangement does not take into account the fact that the Jordan-Wigner transformations strongly depends on the order of the operators. Hence, if we imagine to re-organize the fermionic sites and then invert the Jordan Wigner transformation, we will obtain a spin model completely different from the starting one.

\subsection{Ground state and energies}

The fermionic Hamiltonian in equation (3) can be easily diagonalized performing a Fourier transform in the momentum space where the system can be written as the sum of $N / 2$ non interacting terms

$$
\begin{aligned}
& H_{\phi}^{(n)}=\sum_{k>0} \tilde{H}_{\phi, k}^{(n)} \\
& \tilde{H}_{\phi, k}^{(n)}=2 \imath \delta_{\phi, k}^{(n)}\left(b_{k}^{\dagger} b_{-k}^{\dagger}-b_{-k} b_{k}\right)+2 \varepsilon_{\phi, k}^{(n)}\left(b_{k}^{\dagger} b_{k}+b_{-k}^{\dagger} b_{-k}-1\right) .
\end{aligned}
$$

Each terms $\tilde{H}_{\phi, k}^{(n)}$ act only on fermions with momentum $k$ and/or $-k$ with $k=\pi l / N$ and $l<N$ positive integer. The parameters $\delta_{\phi, k}^{(n)}$ and $\varepsilon_{\phi, k}^{(n)}$ are given by

$$
\begin{aligned}
& \delta_{\phi, k}^{(n)}=J \sin ((n+1) k) \cos \phi, \\
& \varepsilon_{\phi, k}^{(n)}=J(\cos ((n+1) k) \cos \phi+\sin \phi) .
\end{aligned}
$$

Since the Hamiltonian is the sum of terms acting on different Hilbert space, the ground state of the total Hamiltonian will be the tensor product of the ground states of the different $\tilde{H}_{\phi, k}^{(n)}$, i.e.

$$
\left|\psi_{\phi}^{(n)}\right\rangle=\bigotimes_{k}\left|\psi_{\phi, k}^{(n)}\right\rangle
$$




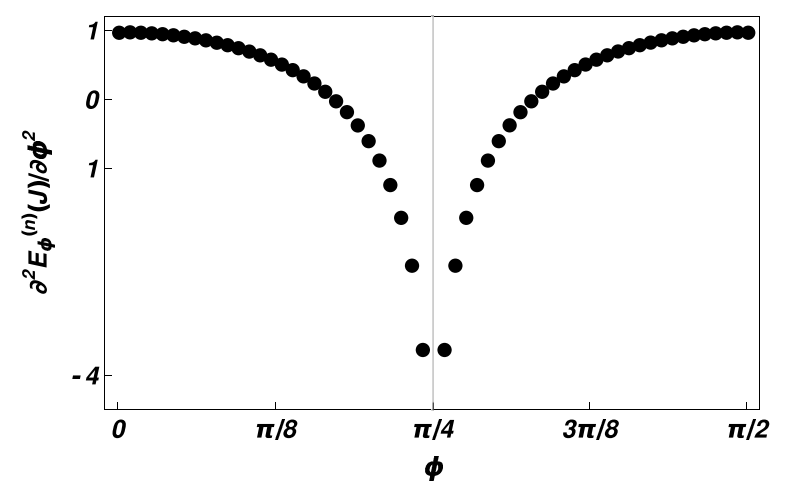

Figure 1. Behavior of the second derivative of the ground state energy density $E_{\phi, k}^{(n)}$, as function of $\phi$, for $n=1$. The divergence is independent of $n$ at the critical value $\phi_{\mathrm{c}}=\frac{\pi}{4}$ and corresponds to a vanishing energy gap between the ground state and the first excited state.

The local ground state $\left|\psi_{\phi, k}^{(n)}\right\rangle$ is given by

$$
\left|\psi_{\phi, k}^{(n)}\right\rangle=\alpha_{\phi, k}^{(n)}\left|1_{k}, 1_{-k}\right\rangle+\beta_{\phi, k}^{(n)}\left|0_{k}, 0_{-k}\right\rangle
$$

with the parameter $\alpha_{\phi, k}^{(n)}$ and $\beta_{\phi, k}^{(n)}$ equal to

$$
\begin{aligned}
& \alpha_{\phi, k}^{(n)}=\imath \frac{\varepsilon_{\phi, k}^{(n)}-E_{\phi, k}^{(n)}}{\sqrt{\left(\delta_{\phi, k}^{(n)}\right)^{2}+\left(\varepsilon_{\phi, k}^{(n)}-E_{\phi, k}^{(n)}\right)^{2}}}, \\
& \beta_{\phi, k}^{(n)}=\frac{\delta_{\phi, k}^{(n)}}{\sqrt{\left(\delta_{\phi, k}^{(n)}\right)^{2}+\left(\varepsilon_{\phi, k}^{(n)}-E_{\phi, k}^{(n)}\right)^{2}}},
\end{aligned}
$$

and an associated energy equal to

$$
E_{\phi, k}^{(n)}=-2 \sqrt{\left(\delta_{\phi, k}^{(n)}\right)^{2}+\left(\varepsilon_{\phi, k}^{(n)}\right)^{2}}
$$

It is extremely relevant to note that while $\alpha_{\phi, k}^{(n)}$ is an imaginary number $\beta_{\phi, k}^{(n)}$ is a real one. This property will play a fundamental role when we will determine the spin correlation functions.

Accordingly with the fact that the ground state of the system is the tensor product of states defined on different Hilbert space, its energy can be would be the sum of the different energies. In the thermodynamic limit, replacing the sum with the integral, the expression of the energy density becomes

$$
E_{\phi}^{(n)}=-\frac{2 J}{\pi} \int_{0}^{\pi} \sqrt{1+\cos ((n+1) k) \sin (2 \phi)} \mathrm{d} k .
$$

The knowledge of the energy density of the ground state allows us to determine the presence of quantum phase transitions. In fact, in agreement with the general theory of continuous phase transitions at zero temperature, the divergence of the second derivative of the energy density, with respect to the Hamiltonian parameter, signals 
the presence of a quantum critical point [5]. In figure 1, we plot the second derivative of the energy density as a function of the parameter $\phi$. We can see that the second derivative of the energy density shows a divergence at $\phi=\phi_{\mathrm{c}} \equiv \pi / 4$, that results to be independent on $n$.

\subsection{Fermionic correlations functions}

From the knowledge of the ground state we can evaluate the spin correlation functions. However, being the ground state in terms of fermionic variables, we have to transform the spin operator associated to the correlation function into a fermionic one. To make this transformations we introduce two different types of Majorana fermionic operators, i.e.

$$
A_{j}=c_{j}+c_{j}^{\dagger} \quad \text { and } \quad B_{j}=c_{j}-c_{j}^{\dagger} .
$$

Any spin operator can be mapped into an ordered products of the two types of Majorana fermionic operators [45]. Moreover, accordingly with the Wick's theorem [46], the expectation value of the tensor product of fermionic operators can be expressed in terms of the contractions involving only one- and two-body correlation functions. Considering the parity symmetry of the Hamiltonian, and hence of the ground state, the expectation value of a single Majorana operator vanish, i.e. $\left\langle A_{i}\right\rangle=\left\langle B_{i}\right\rangle=0$ (here and in the following $\langle\mathcal{O}\rangle$ is a shortcut for $\left.\left\langle\psi_{\phi}^{(n)}|\mathcal{O}| \psi_{\phi}^{(n)}\right\rangle\right)$. Moreover taking into account that in equation (8) one of the two coefficients is real and the second is imaginary it is easy to prove that $\left\langle A_{i} A_{k}\right\rangle=-\left\langle B_{i} B_{k}\right\rangle=\delta_{i k}$

These results play a key role when we evaluate the spin correlation functions and, consequently, of the entanglement measures. Indeed, at first, if a spin operator is mapped into an odd number of fermionic operators, its expectation value on the ground state of the system has to vanish. In fact, accordingly with the Wick's theorem, it can be written as a linear combination of terms each one of them include the expectation value of a single Majorana operator that it is always zero. This is the case of spin correlation functions which operator does not commute with the parity along $z$, i.e. $P_{z}=\bigotimes_{i} \sigma_{i}^{z}$. Moreover, also a spin operator that are mapped in fermionic one with a different number of $A_{i}$ and $B_{i}$ operators has to vanish due to the fact that $\left\langle A_{i} A_{k}\right\rangle=\left\langle B_{i} B_{k}\right\rangle=0$. This is the case of operators with am odd number of $\sigma_{i}^{x}$ or $\sigma_{i}^{y}$ operators which correlation function vanishes, independently on the set of Hamiltonian parameters, even if the operator commutes with the parity operator along the $z$ spin direction. Therefore the only spin correlation functions that are not identically zero are those that can be written in terms of functions $G_{i, k}(n, \phi) \equiv\left\langle B_{i} A_{k}\right\rangle$.

The functions $G_{i, k}(n, \phi)$ depends on the two sites on which they are evaluated. However, because we are considering models that are invariant under spatial translation, they have to depend only on the relative distance $r=i-k$ and not on the precise choice of the site $i$ and $k$, i.e. $G_{i, k}(n, \phi)=G_{r}(n, \phi)$. With a small algebra, taking into account equations (6) and (7), in the thermodynamic limit, we obtain for $G_{r}(n, \phi)$ the following expression

$$
G_{r}(n, \phi)=\frac{1}{\pi} \int_{0}^{\pi} \frac{\cos (k(n+1+r)) \cos \phi-\cos (k r) \sin \phi}{\sqrt{1+\cos ((n+1) k) \sin (2 \phi)}} \mathrm{d} k .
$$


Defining $I(p)$ as

$$
I(p)=\frac{1}{2 \pi} \int_{0}^{2 \pi} \frac{\cos (k p)}{\sqrt{1-\sin (2 \phi) \cos (k(n+1))}} \mathrm{d} k
$$

the fermionic correlation functions $G_{r}(n, \phi)$ can be written as

$$
G_{r}(n, \phi)=\cos (\phi) I(n+1+r)+\sin (\phi) I(r) .
$$

The introduction of the function $I(p)$ allows us to prove an important properties of $G_{r}(n, \phi)$. In fact, taking into account the periodicity of the integrand in the equation (13), we have that $I(p)$ can be rewritten as

$$
I(p)=\frac{1}{2 \pi} \sum_{l=0}^{n} \int_{0}^{\frac{2 \pi}{n+1}} \frac{\cos (k p) \cos \left(\frac{2 \pi l p}{n+1}\right)-\sin (k p) \sin \left(\frac{2 \pi l p}{n+1}\right)}{\sqrt{1-\sin (2 \phi) \cos (k(n+1))}} \mathrm{d} k .
$$

But the $\operatorname{sum} \sum_{l=0}^{n}\left(\cos (k p) \cos \left(\frac{2 \pi l p}{n+1}\right)-\sin (k p) \sin \left(\frac{2 \pi l p}{n+1}\right)\right)$ is non zero if and only if $p=(n+1) m$ where $m$ is an integer (positive or negative). Hence $I(p)$ is non zero if and only if $p=(n+1) m$. Consequently also $G_{r}(n, \phi)$ is non vanishing if and only if $r=m(n+1)$ where $m$ in an integer.

\subsection{Spin correlations functions}

From the knowledge of the $G_{r}(n, \phi)$ we can evaluate all the correlation functions. Let us make some consideration about the main ones. To begin, let us consider the magnetization along $z$ that it is equal to $\left\langle\sigma_{i}^{z}\right\rangle=-G_{0}(n, \phi)$. The presence of the external field along the $z$ axis induces, on the contrary of the models in [23] a non vanishing magnetization that is always different from zero, for all possible values of $\phi \neq 0$ and for all $n$. Moreover, in the same direction, the two-body correlation function can be written as

$$
\left\langle\sigma_{i}^{z} \sigma_{i+r}^{z}\right\rangle=G_{0}^{2}(n, \phi)-G_{r}(n, \phi) G_{-r}(n, \phi),
$$

and hence for $r \neq m(n+1)$ it factorizes in the product of the two magnetizations, i.e. $\left\langle\sigma_{i}^{z} \sigma_{i+r}^{z}\right\rangle=\left\langle\sigma_{i}^{z}\right\rangle^{2} \forall r \neq m(n+1)$.

On the other side the expression of the correlation functions $\left\langle\sigma_{i}^{x} \sigma_{i+r}^{x}\right\rangle$ and $\left\langle\sigma_{i}^{y} \sigma_{i+r}^{y}\right\rangle$ are more complex. With some algebra [45] it is easy to prove that they can be written, in terms of the $G_{r}(n, \phi)$, respectively as the determinant of the following matrices

$$
\begin{gathered}
\left(\begin{array}{cccc}
G_{-1}(n, \phi) & G_{-2}(n, \phi) & \cdots & G_{-r}(n, \phi) \\
G_{0}(n, \phi) & G_{-1}(n, \phi) & \cdots & G_{1-r}(n, \phi) \\
\vdots & \vdots & \ddots & \vdots \\
G_{r-2}(n, \phi) & G_{r-3}(n, \phi) & \cdots & G_{-1}(n, \phi)
\end{array}\right) \\
\left(\begin{array}{cccc}
G_{1}(n, \phi) & G_{2}(n, \phi) & \cdots & G_{r}(n, \phi) \\
G_{0}(n, \phi) & G_{1}(n, \phi) & \cdots & G_{r-1}(n, \phi) \\
\vdots & \vdots & \ddots & \vdots \\
G_{2-r}(n, \phi) & G_{3-r}(n, \phi) & \cdots & G_{1}(n, \phi)
\end{array}\right) .
\end{gathered}
$$




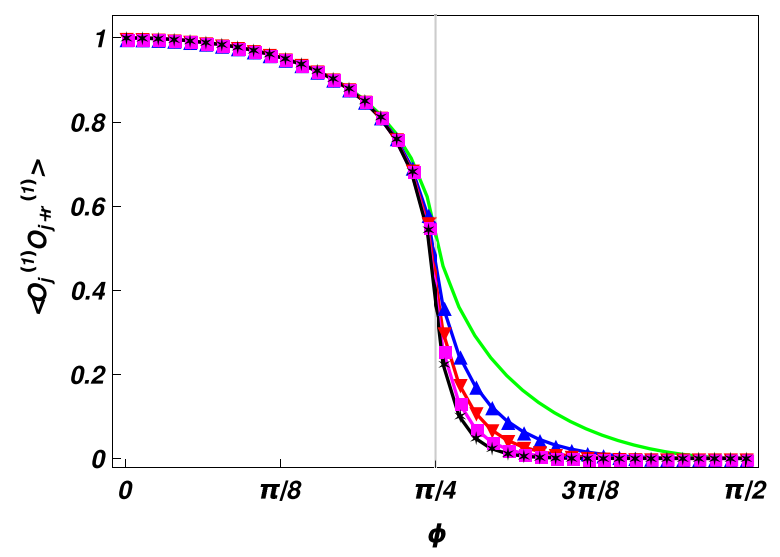

Figure 2. Behavior of the expectation value $\left\langle\mathcal{O}_{j}^{(n)} \mathcal{O}_{j+r}^{(n)}\right\rangle$, for $n=1$ and $r=3,6,9,12,15$, as a function of the phase parameter $\phi$ : green dots (upper curve) $r=3$, blue up-triangles $r=6$, red down-triangles $r=9$, magenta squares $r=12$ and black stars (lower curve) $r=15$. As $r$ increases, the expectation value tends to disappear in the paramagnetic phase while it remains finite in the cluster phase.

Taking into account that the fermionic correlation functions $G_{r}(n, \phi)$ for all $r \neq l(n+1)$ vanish, it is easy to prove that

$$
\left\langle\sigma_{i}^{x} \sigma_{i+r}^{x}\right\rangle=\left\langle\sigma_{i}^{y} \sigma_{i+r}^{y}\right\rangle=0 .
$$

Instead for $r=l(n+1)$ both the two correlation functions are different from zero and in the very relevant case in which $l=1$ and hence $r=n+1$, we obtain that

$$
\begin{aligned}
\left\langle\sigma_{i}^{x} \sigma_{i+n+1}^{x}\right\rangle & =(-1)^{n} G_{-(n+1)}(n, \phi) G_{0}(n, \phi)^{n}, \\
\left\langle\sigma_{i}^{y} \sigma_{i+n+1}^{y}\right\rangle & =(-1)^{n} G_{(n+1)}(n, \phi) G_{0}(n, \phi)^{n} .
\end{aligned}
$$

In the limiting case of the quantum Ising model in the transverse field that we obtain setting $n=0$, the equation (19) reduces at the well known expression of the spin correlation functions obtained in [45].

\section{Phase diagrams and order parameters}

As we have seen in section 2.1, the presence of a divergence in the second derivative of the ground state energy density signals that, regardless the value of $n$, in the thermodynamic limit the models undergo to a quantum phase transition at $\phi=\phi_{\mathrm{c}} \equiv \pi / 4$. However, the presence of such divergence does not provide any information about the kind of phases that are realized in the system before and after the quantum critical point. To make this analysis let us rewrite the Hamiltonian in equation (1) by using well tailored cluster operators. Such operators, that we named $\mathcal{O}_{j}^{(n)}$ are defined as

$$
\mathcal{O}_{j}^{(n)}=\left\{\begin{array}{ll}
\left(\bigotimes_{k=1}^{j-n-1} \sigma_{k}^{z}\right) \sigma_{j-n}^{y} \sigma_{j-n+1}^{x} \cdots \sigma_{j-1}^{y} \sigma_{j}^{x} & \text { odd } n \\
\sigma_{j}^{x} \sigma_{j+1}^{y} \sigma_{j+2}^{x} \cdots \sigma_{j+n}^{x} & \text { even } n
\end{array} .\right.
$$




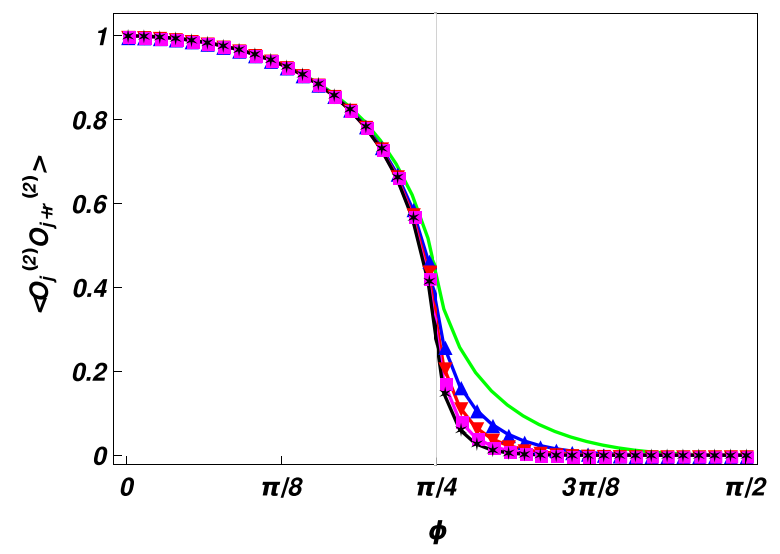

Figure 3. Behavior of the expectation value $\left\langle\mathcal{O}_{j}^{(n)} \mathcal{O}_{j+r}^{(n)}\right\rangle$, for $n=2$ and $r=3,6,9,12,15$, as a function of the phase parameter $\phi$ : green dots (upper curve) $r=3$, blue up-triangles $r=6$, red down-triangles $r=9$, magenta squares $r=12$ and black stars (lower curve) $r=15$. As $r$ increases, the expectation value tends to disappear in the paramagnetic phase while it remains finite in the cluster phase.

The main difference between the two different cases, i.e. even and odd $n$, is that the operators $\mathcal{O}_{j}^{(n)}$ are defined, when $n$ is even, on a finite support made by $n+1$ contiguous spins while for odd $n$ the dimension of support depends on the site and in the thermodynamic limit diverges with $N$. However, regardless to $n$, the operators $\mathcal{O}_{j}^{(n)}:(1)$ satisfy the commutation relation $\left[\mathcal{O}_{j}^{(n)}, \mathcal{O}_{k}^{(n)}\right]=0 \forall j, k$; (2) admit two different eigenvalues, namely \pm 1 , both of them with a degeneracies equal to $2^{n}$; (3) do not commute with the parity along $z$.

By using these operators we can rewrite the Hamiltonian of equation (1) as

$$
H_{\phi}^{(n)}=-J \cos (\phi) \sum_{j} \mathcal{O}_{j}^{(n)} \mathcal{O}_{j+1}^{(n)}+J \sin (\phi) \sum_{j} \sigma_{j}^{z}
$$

Let us set, for a moment, $\phi=0$. In this case, taking into account the commutation relation $\left[\mathcal{O}_{j}^{(n)}, \mathcal{O}_{k}^{(n)}\right]=0$, the Hamiltonian will admit several factorized ground states, all of them characterized by the fact that $\left\langle\mathcal{O}_{j}^{(n)} \mathcal{O}_{j+1}^{(n)}\right\rangle=1$ and $\left\langle\mathcal{O}_{j}^{(n)}\right\rangle= \pm 1$. Taking into account that $\mathcal{O}_{j}^{(n)}$ does not commute with the parity, the fact that $\left\langle\mathcal{O}_{j}^{(n)}\right\rangle= \pm 1$ signals the presence of an ordered phase and makes the expectation value of the operator $\mathcal{O}_{j}^{(n)}$ the main candidate to play the role of the order parameter.

This picture is strengthened if we look at figures 2 ( $n$ odd) and 3 ( $n$ even) in which we plot the expectation values of $\left\langle\mathcal{O}_{j}^{(n)} \mathcal{O}_{j+r}^{(n)}\right\rangle$ as a function of $\phi$, by varying $r$. From the two figures, we observe that, as $r$ increases, these expectation values tend to disappear for $\phi>\phi_{\mathrm{c}}$, while they remain finite in the cluster phase, making

$$
\left\langle\mathcal{O}_{j}^{(n)}\right\rangle=\lim _{r \rightarrow \infty} \sqrt{\left\langle\mathcal{O}_{j}^{(n)} \mathcal{O}_{j+r}^{(n)}\right\rangle}
$$

an excellent order parameter to describe the kind of phase realized in the system. 


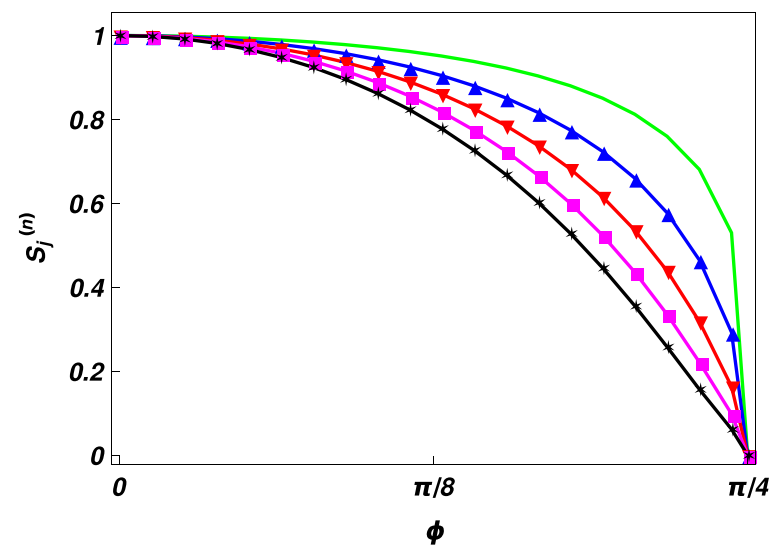

Figure 4. Behavior of the string order parameter $S_{j}^{(n)}$, for several $n$, as a function of the phase parameter $\phi$ : green dots (upper curve) $n=1$; blue up-triangles $n=3$; red down-triangles $n=5$; magenta squares $n=7$; black stars (lower curve) $n=9$. The dots represent the numerical results whereas the curves correspond to the behavior of the string order parameter $S^{(n)}$ defined in equation (23).

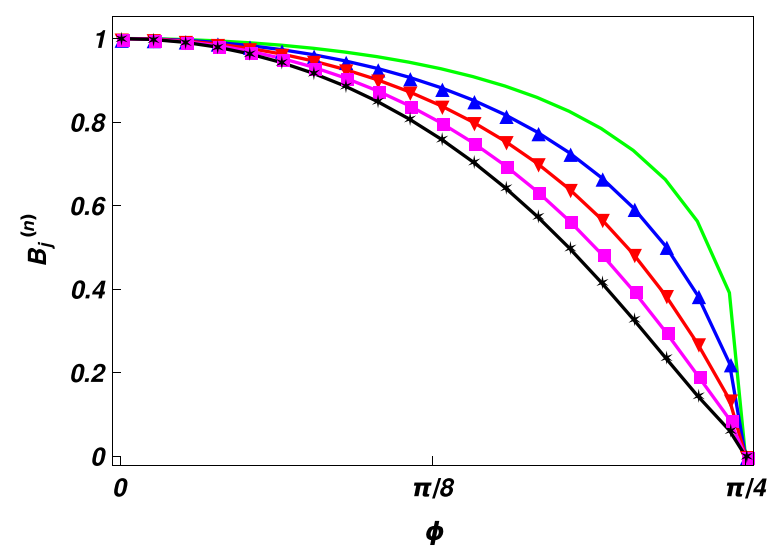

Figure 5. Behavior of the nematic order parameter $B_{j}^{(n)}$, for several $n$, as a function of the phase parameter $\phi$ : green dots (upper curve) $n=2$; blue up-triangles $n=4$; red down-triangles $n=6$; magenta squares $n=8$; black stars (lower curve) $n=10$. The dots represent the numerical results whereas the curves correspond to the behavior of the nematic order parameter $B^{(n)}$ defined in equation (23).

However, as we saw in equation (20), passing from even $n$ to odd one the structure of the $\mathcal{O}_{j}^{(n)}$ operators changes significantly. This implies that, depending on the parity of $n$, we have two different kinds of order. When $n$ is even we have the so-called nematic order (which behavior for several $n$ is shown in figure 5), that is an order that can be seen as the generalization of the standard magnetic order usually founded in spin models with interaction between neighboring spins as the Ising model. As for the standard magnetic order, the presence of a nematic order implies the existences of ground states that can brake at least one symmetry of the Hamiltonian. Both of them can be characterized by an order parameter that has a finite support but, while in the magnetic order it coincides with a single spin, in the nematic case the support is strictly greater than one. On the other hand, when $n$ is odd we have that in the system a 
symmetry protected topological order is realized (which behavior for several $n$ is shown in figure 4). As in the standard topological order, that cannot be realized in a 1D spin$1 / 2$ model [47], two distinct topological states cannot be smoothly deformed into each other without a phase transition, if the deformation preserves the symmetry. However, differently from the true topological ordered phase they all can be smoothly deformed into the same trivial product state without a phase transition, if the symmetry is broken during the deformation.

Analyzing the numerical data obtained for both order parameters $\mathcal{S}_{j}^{(n)}$ and $\mathcal{B}_{j}^{(n)}$, we find finally the same dependence on $n$ and $\phi$, i.e.

$$
\begin{aligned}
& \mathcal{S}^{(n)}=\left(1-\tan (\phi)^{2}\right)^{\frac{n+1}{8}}, \\
& \mathcal{B}^{(n)}=\left(1-\tan (\phi)^{2}\right)^{\frac{n+1}{8}}
\end{aligned}
$$

from which we deduce the critical exponent $\beta$

$$
\beta=\beta(n)=\frac{n+1}{8} .
$$

The fact that the critical exponent $\beta$ depends on $n$ point out that the models under analysis fall, depending on $n$, into different classes of symmetries. For $n=0$ we recover, as expected, the critical exponent for the Ising class of symmetries $\left(\mathbb{Z}_{2}\right)$ and also the block order parameter becomes equal to $\left\langle\sigma_{i}^{x}\right\rangle$, i.e. the standard magnetic order parameter that signals the presence of an ordered phase for that class of models. To determine the classes of symmetries for the model with $n>0$ is more complicated because we have to analyze each single case separately. However as we will see better in section for $n=2$ we can provide a simple answer using the analogy with the cluster-Ising model analyzed in [37].

On the contrary, above the quantum critical point, i.e. $\phi>\phi_{\mathrm{c}}$, the system is dominated by the external magnetic field. In such a phase, there is no order parameter and the system is in a typical paramagnetic phase.

\section{The entanglement properties}

Let us now begin to analyze the different entanglement properties of our family of models. We focus on two different kind of entanglement. The first is the entanglement between different spins in a block of the chain, the second is the entanglement between the block of spins and the rest of the system. The starting point to analyze both of them is the reduced density matrix obtained, from the ground state, tracing out all the degrees of freedom of the spins outside the block of interest. Such reduced density matrix can be written in terms of the $m$-point spin correlation functions [48] as

$$
\rho_{m}^{(n)}=\frac{1}{2^{m}} \sum_{\alpha_{1}, \ldots, \alpha_{m}}\left\langle\sigma_{1}^{\alpha_{1}} \sigma_{2}^{\alpha_{2}} \cdots \sigma_{m}^{\alpha_{m}}\right\rangle \sigma_{1}^{\alpha_{1}} \sigma_{2}^{\alpha_{2}} \cdots \sigma_{m}^{\alpha_{m}},
$$




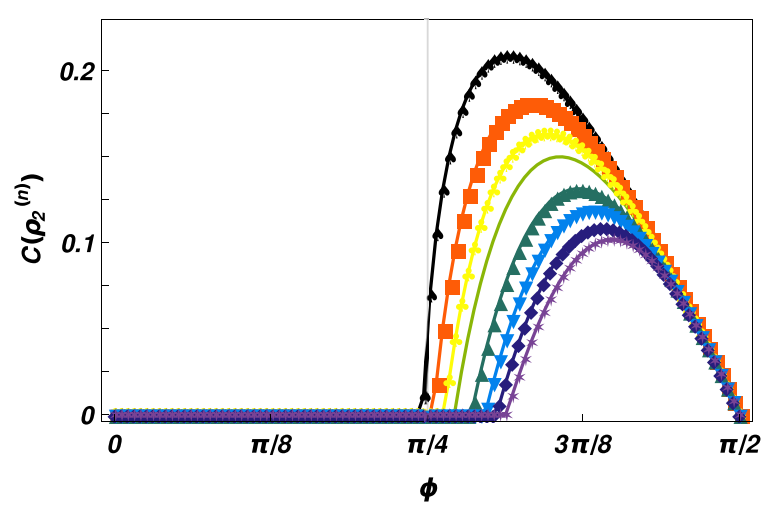

Figure 6. Dependence of the concurrence $C\left(\rho_{2}^{(n)}\right)$ as function of the phase parameter $\phi$, for $r=n+1$ and different $n$ that runs from 1 (highest curve) to 8 (lowest curve). Note that only for $n=1$ concurrence is non-zero before the critical point.

where $\alpha_{i}=0, x, y, z$ and $\sigma_{i}^{0}$ denotes the identity matrix.

\subsection{Entanglement between two spins}

For what concerns the entanglement between two spins in a block, we can prove the following theorem:

Theorem 1. If the distance $r$ between the two spins is not an integer multiple of $n+1$ the two spins are not entangled.

Proof. To proof the theorem is it enough to recall the results obtained in section 2.3, for the spins correlation functions. In fact, in agreement with equation (25), the 2-spin reduced density matrix can be written as a linear composition of single-body and twobody spin correlation functions. For what concern the single-body correlation functions, we have that $\left\langle\sigma_{i}^{x}\right\rangle=\left\langle\sigma_{i}^{y}\right\rangle=0$, because of the properties of the Majorana fermionic operators $\left\langle A_{i}\right\rangle=\left\langle B_{i}\right\rangle=0$. On the other hand, for what concern the two-body correlation functions, all the functions that involve different spin operators vanish in agreement with the fact that $\left\langle A_{i}\right\rangle=\left\langle B_{i}\right\rangle=0$ and $\left\langle A_{i} A_{j}\right\rangle=\left\langle B_{i} B_{j}\right\rangle=\delta_{i, j}$. Thus, the two spin reduced density matrix depends on four different correlation functions only: $\left\langle\sigma_{i}^{z}\right\rangle,\left\langle\sigma_{i}^{x} \sigma_{i+r}^{x}\right\rangle$, $\left\langle\sigma_{i}^{y} \sigma_{i+r}^{y}\right\rangle$ and $\left\langle\sigma_{i}^{z} \sigma_{i+r}^{z}\right\rangle$. However, as we have proved in section 2.3 if $r \neq l(n+1)$ where $l$ is an integer than $\left\langle\sigma_{i}^{x} \sigma_{i+r}^{x}\right\rangle=\left\langle\sigma_{i}^{y} \sigma_{i+r}^{y}\right\rangle=0$. Hence the reduced density matrix depends only on $\left\langle\sigma_{i}^{z}\right\rangle$ and $\left\langle\sigma_{i}^{z} \sigma_{i+r}^{z}\right\rangle$ and therefore is diagonal in the base of the eigenstates of $\sigma_{i}^{z}$ and $\sigma_{i+r}^{z}$. But this base is made by state that are tensor product of states defined on a single spin. Being diagonal in a base made by states that are tensor product of local states, the reduced density matrix cannot be entangled. QED

On the contrary, when $r=l(n+1)$, since $\left\langle\sigma_{i}^{x} \sigma_{i+r}^{x}\right\rangle \neq 0$ and $\left\langle\sigma_{i}^{y} \sigma_{i+r}^{y}\right\rangle \neq 0$, and hence there can be a region of the Hamiltonian parameters for which the two spins are entangled. We quantify such entanglement in terms of the concurrence $C\left(\rho_{2}^{(n)}\right)[42,49]$. In figure 6 , we plot $C\left(\rho_{2}^{(n)}\right)$ as a function of the phase parameter $\phi$, for $l=1$ and hence $r=n+1$ for several $n$. Regardless $n$ the different concurrences show a similar behavior. With the single exception of $n=1$ they are different from zero in a region confined in 
the paramagnetic phase, increase up to a maximum value, and vanishes at $\phi=\pi / 2$ where all the systems admit a factorization point [16-18]. Increasing $n$, the concurrence becomes smaller and smaller and the relative maximum goes towards higher value of $\phi$. On the contrary, for all $l>1$ numerical evaluation shows that all the concurrences are identically zero. Therefore, the entanglement is always limited between the spins at the end points of a cluster term.

It is interesting to compare the behavior of the concurrences between the two spins at the ends of the cluster term in our model with the one, well known, between neighbors spins of an Ising model in the transverse field [2, 3, 48]. The comparison shows some similarity but also some relevant differences. For what concern the similarity, we have that also the concurrence between next neighbors spins in the quantum Ising model is characterized by a local maximum in the paramagnetic phase just before the phase transition and by an annulment at the factorization point at $\phi=\pi / 2$. But in the magnetic phase of the Ising model, which takes the place of the cluster phase because the cluster term becomes a normal two-body interaction, is always characterized, with the exception of $\phi=0$ (factorization point of the magnetically ordered phase) by a non-zero concurrence. Moreover, even if only in a small region of the parameters around the critical point, the Ising model has also a non-zero entanglement between next neighbors spins that has no equivalent in the models with $n \geqslant 1$ [2].

\subsection{Genuine multipartite entanglement}

For what concern the genuine multipartite entanglement, we prove the following theorem:

Theorem 2. For each block made by $m$ adjacent spins, with $m \leqslant n+2$, there is no genuine multipartite entanglement.

Proof. The proof is based on the fact that, following the definition of the genuine multipartite entanglement for a mixed state, it must be impossible to find a decomposition of the reduced density matrix in states that show only entanglement between a couple of spins.

To start our proof, let us consider a block made by $m=n+2$ adjacent spins. The reduced density matrix on such a block can be written in terms of the spin correlation functions and, in turn, all the spin correlation functions must be written in terms of the $G_{r}(n, \phi)$ functions. Taking into account the results of section 2.2 and the fact that the maximum distance between the two spins in the block is $n+1$, the reduced density matrix depends only on three different fermionic correlation functions: $G_{0}(n, \phi), G_{n+1}(n, \phi)$ and $G_{-n-1}(n, \phi)$. Therefore, the only spin correlation functions different from zero are the ones that are diagonal in the natural base or that are associated to an inversion of the two spins at the end of the block. Hence, in the natural base, the reduce density matrix can be written as a convex combination

$$
\rho_{n+2}^{(n)}=\sum_{i} p_{i}\left(\bigotimes_{k=2, n+1} \chi_{i, k}\right) \bigotimes \chi_{i, 1, n+2},
$$


where $\chi_{i, k}$ is a state defined on the $k$ th spin of the block and $\chi_{i, 1, n+2}$ is a state (entangled or not) defined on the two endpoints of the block. In other words, the reduced density matrix can be written as a linear combination of states that, with the only exception of a possible bipartite entanglement between the two endpoints of the block, are fully factorized. In such state it comes immediately that any multipartite entanglement has to vanish.

If now we consider a block made by $m=n+1$ adjacent spins, we have that the reduced density matrix can be obtain by equation (26) tracing out one of the two endpoint spins. Thus, we obtain a reduced density matrix that is a linear convex combination of fully disentangled states and hence it does not admit any entanglement. continuing this reasoning we can prove that also any subsystem made by $m<n+1$ spins cannot show multipartite entanglement. QED

Because of the well known problem of the lack of a general closed formula for the genuine multipartite entanglement, it is impossible to prove analytically that such absence of genuine multipartite entanglement extends also at subsets made by more than $m+2$ spins. To flank this problem we made some numerical simulations, based on the criterion developer in [24, 25]. Unfortunately, this criterion, like others developed with the same goal, is sufficient but not necessary. In other words, if the criterion is verified, we have a proof of the presence of genuinely multipartite entanglement, but if it is not verified, the criterion does not provide any answer. In all our numerical simulations with several $m \geqslant n+2$ with $n \geqslant 1$ the criterion has never been verified. This leads us to think that the absence of multipartite entanglement also extends to blocks larger than $n+2$.

It is interesting to make a comparison with the results reported in [23], for the $n$-cluster Ising models, where, on the contrary, there is no bipartite entanglement but a significant value of genuine multipartite entanglement evaluated with the so called generalized concurrence [50-52]. Comparing these two results, and taking into account the proof of the presence of genuine multipartite entanglement in the $x y$ model $[20,21]$, we may counter-intuitively conclude that a fundamental requirement to have multipartite entanglement is the presence, in the Hamiltonian, of a simple Ising-like interaction. The presence of a cluster interaction decreases the total value of the entanglement in the system and allows to separate the bipartite and multipartite contributions in a way that has no equivalent with the more common two-body interactions. This opens up countless applications, for cluster models, in the field of quantum computing. To give an example, the fact that, setting $n=1$, in our models the bipartite entanglement extends also to cluster phase and the three-partite entanglement make the same in the cluster-Ising model [22], open the possibility to convert bipartite to multipartite entanglement, and vice versa, using the adiabatic deformation of the ground state [54].

\subsection{Block entanglement}

Let us now analyze the entanglement between a block of $m$ adjacent spins and the rest of the chain in the quantum critical point $\phi=\pi / 4$ that is related to the holomorphic and anti-holomorphic sectors in conformal field theory [10]. 


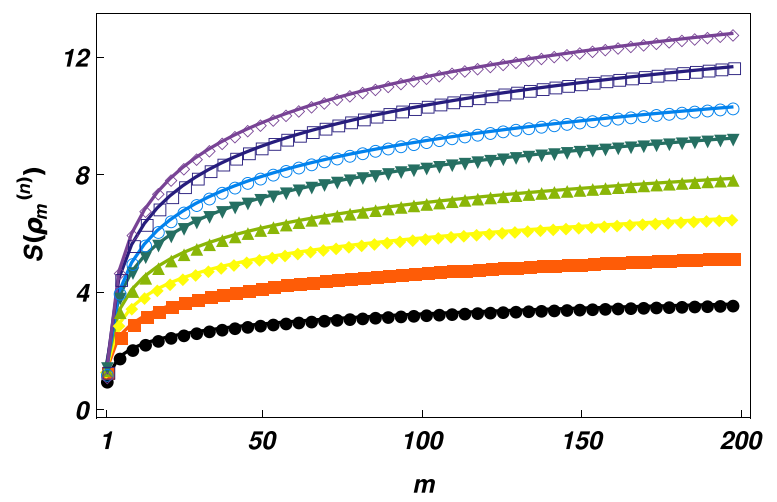

Figure 7. Behavior of the von Neumann entropy $S\left(\rho_{m}^{(n)}\right)$, as a function of the size $m$ of the block, for different values of size $n+2$ of the cluster. The value of $n$ runs from 1 (lowest black curve) to 8 (highest violet curve). Independently of $n$, the von Neumann entanglement entropy diverges, at a quantum critical point $\phi_{\mathrm{c}}=\pi / 4$, as a logarithmic function of $m$.

For this purpose, we compute the von Neumann entanglement entropy [53] of the reduced density matrix of a block of $m$ spins

$$
S\left(\rho_{m}^{(n)}\right)=-\operatorname{Tr}\left[\rho_{m}^{(n)} \log _{2}\left(\rho_{m}^{(n)}\right)\right] .
$$

Using the methods developed in [7, 8], we find

$$
S\left(\rho_{m}^{(n)}\right)=\sum_{j=1}^{m} H_{\text {Shannon }}\left(\frac{1+\nu_{j}^{(n)}}{2}\right),
$$

where $H_{\text {Shannon }}(x)$ is the Shannon entropy

$$
H_{\text {Shannon }}(x)=-x \log _{2}(x)-(1-x) \log _{2}(1-x),
$$

and $\nu_{j}^{(n)}$ are the imaginary part of the eigenvalues of the matrix

$$
\left(\Gamma^{(n)^{\prime}}\right)_{i j}=\delta_{i j}-\imath\left(\Gamma_{m}^{(n)}\right)_{i j}
$$

with

$$
\Gamma_{m}^{(n)}=\left(\begin{array}{cccc}
\Pi_{0}^{(n)} & \Pi_{-1}^{(n)} & \cdots & \Pi_{-m+1}^{(n)} \\
\Pi_{1}^{(n)} & \Pi_{0}^{(n)} & \cdots & \Pi_{-m+2}^{(n)} \\
\vdots & \vdots & \ddots & \vdots \\
\Pi_{m-1}^{(n)} & \Pi_{m-2}^{(n)} & \cdots & \Pi_{0}
\end{array}\right)
$$

and

$$
\Pi_{r}^{(n)}=\left(\begin{array}{cc}
0 & G_{r}(n, \phi) \\
-G_{-r}(n, \phi) & 0
\end{array}\right) .
$$


We evaluate numerically the von Neumann entanglement entropy as a function of the size of the block, at the critical point $\phi=\phi_{\mathrm{c}}$ (see figure 7). Analyzing the data, we deduce that the von Neumann entropy obeys to the following logarithmic law

$$
S\left(\rho_{m}^{(n)}\right) \simeq 0.17(1+n) \log _{2} m+\operatorname{const}(n) .
$$

The multiplicative constant in front of the logarithmic term is known to be related to the central charge of the $1+1$ dimensional conformal field theory, that describes the critical behavior of the chain via the relation [6]

$$
S_{m}=\frac{\mathrm{c}+\overline{\mathrm{c}}}{6} \log _{2} m
$$

where $c$ and $\bar{c}$ are the central charges of the so-called holomorphic and anti-holomorphic sectors [10]. Due to the existence of a duality in the system, we have that $\mathrm{c}=\overline{\mathrm{c}}$ and hence

$$
\mathrm{c}=\mathrm{c}(n) \simeq 0.51(1+n)
$$

As for the $\beta$ critical exponent analyzed in section 3, two quantum 1D systems belonging in the same universality class have the same central charge. Hence the fact that the central charge of the $n$-cluster models in a transverse magnetic field depends on $n$, implies that they fall into different universality classes. Taking into account the unavoidable approximations of the numerical approach, the result reported in equation (35) for $n=0$ is compatible with the central charge obtained for the Ising model that is equal to $\frac{1}{2}$. Moreover settings $n=2$ we obtain a central charge equal to $\mathrm{c}=1.51$ and from equation (24) a critical exponent $\beta=\frac{3}{8}$. These two values are compatible with the ones obtained in [37] for the cluster-Ising model (respectively $\mathrm{c}=\frac{3}{2}$ and $\beta=\frac{3}{8}$ ). Hence the cluster-Ising model and the 2 -cluster model in the external field lives in the same universality class that is the one characterized by a $\mathbb{Z}_{2} \times \mathbb{Z}_{2}$ symmetry [37].

\section{Conclusions}

In summary, we have analyzed a family of fully analytically solvable models, named $n$-cluster models in a transverse magnetic field. These models are characterized by a many body cluster interaction competing with a spatially uniform transverse magnetic field. Using the Jordan-Wigner transformations, we diagonalized the models and proved that their classes of symmetry depends on $n$. For $n=0$ our system reduces to a quantum Ising model and it falls in the $\mathbb{Z}_{2}$ symmetry class, while for $n=2$ the system lives in the $\mathbb{Z}_{2} \times \mathbb{Z}_{2}$ universality class. Regardless the value of $n$, a phase transition always occurs exactly when both interactions are equally weighted. The paramagnetic phase, realized for $\phi>\phi_{\mathrm{c}}$, shows a very similar behavior for all $n$. On the contrary, the cluster phase, realized for $\phi<\phi_{c}$, exhibits two different orders, depending on $n$. For odd (even) $n$, we have a symmetry-protected topological ordered phase (nematic ordered phase) in perfect agreement with the results obtained in [23]. In the case in which $n=0$ the model reduces to a standard Ising model and the nematic parameters 
reduces to the standard magnetic one. The presence of these two important novel phases candidate the $n$-cluster model in the external field as an interesting toy model to study hot topics of the current research, such as the presence of global entanglement [55] or the effects of a sudden quench of the Hamiltonian parameters [19, 56].

In complete contrast with the results obtained for the $n$-cluster Ising models, any multipartite entanglement seems to vanish, while the bipartite entanglement, quantified in terms of the concurrence between two spins at a distance $n+1$, is different from zero in a region that is confined, with the only exception of $n=1$, in the paramagnetic phase. The fact that for $n=1$ a non vanishing concurrence can be found also in the symmetry protected topological phase is extremely relevant. In fact, taking into account that also in the cluster-Ising model [22] the three partite entanglement is non zero in the topological phase it is possible to convert bipartite entanglement into genuine three-partite entanglement, and vice versa, using adiabatic deformation of the ground stats. Moreover, our family of solvable models admits, for large $n$, a very large central charge and hence can be used to test the accuracy of the conformal block expansion [57].

This family of models can be generalized with respect to higher dimensions, both in space and degrees of freedom, and may become a prototype for studying the possible applications of quantum information tasks.

\section{Acknowledgments}

We wish to thank M Dalmonte for the interesting discussions. GZ thanks EQuaMEmulators of Quantum Frustrated Magnetism, Grant Agreement No. 323714 while SMG acknowledges support by the H2020 CSA Twinning project No. 692194, "RBIT-WINNING" and financial support from the Ministry of Science, Technology and Innovation of Brazil.

\section{References}

[1] Venuti L C, Boschi C D E, Morandi G, Roncaglia M and Scaramucci A 2006 Phys. Rev. A 73010303

[2] Osterloh A, Amico L, Falci G and Fazio R 2002 Nature 416608

[3] Amico L, Fazio R, Osterloh A and Vedral V 2008 Rev. Mod. Phys. 80517

[4] Barber M N 1983 Phase Transitions and Critical Phenomena vol 8, ed C Domb and J L Leibovitz (London: Academic)

[5] Sachdev S 2011 Quantum Phase Transitions (Cambridge: Cambridge University Press)

[6] Holzhey C, Larsen F and Wilczek F 1994 Nucl. Phys. B 424443

[7] Vidal G, Latorre J I, Rico E and Kitaev A 2003 Phys. Rev. Lett. 90227902

[8] Latorre J I, Rico E and Vidal G 2004 Quantum Inf. Comput. 4048

[9] Korepin V E 2004 Phys. Rev. Lett. 92096402

[10] Calabrese P and Cardy J 2004 J. Stat. Mech. P06002

[11] Jin B-Q and Korepin V E 2004 J. Stat. Phys. 11679

[12] Eisert J, Cramer M and Plenio M B 2010 Rev. Mod. Phys. 82277

[13] Wen X G and Niu Q 1990 Phys. Rev. B 419377

[14] Wen X G 1990 Int. J. Mod. Phys. B 4239

[15] Chen X, Gu Z-C and Wen X G 2010 Phys. Rev. B 82155138

[16] Giampaolo S M, Adesso G and Illuminati F 2008 Phys. Rev. Lett. 100197201

[17] Giampaolo S M, Adesso G and Illuminati F 2009 Phys. Rev. B 79224434

[18] Giampaolo S M, Adesso G and Illuminati F 2010 Phys. Rev. Lett. 104207202 
[19] Zonzo G, Capolupo A and Giampaolo S M 2017 arXiv:1712.03051

[20] Giampaolo S M and Hiesmayr B C 2013 Phys. Rev. A 88052305

[21] Hofmann M, Osterloh A and Gühne O 2014 Phys. Rev. B 89134101

[22] Giampaolo S M and Hiesmayr B C 2014 New J. Phys. 16093033

[23] Giampaolo S M and Hiesmayr B 2015 Phys. Rev. A 92012306

[24] Huber M, Mintert F, Gabriel A and Hiesmayr B C 2010 Phys. Rev. Lett. 104210501

[25] Gabriel A, Hiesmayr B C and Huber M 2010 Quantum Inf. Comput. 100829

[26] Andreev A F and Grishchuk I A 1984 Sov. Phys.-JETP 60267

[27] Lacroix C, Mendels P and Mila F 2011 Introduction to Frustrated Magnetism (Solid-State Sciences vol 164) (Berlin: Springer)

[28] Martin-Delgado M A, Shankar R and Sierra G 1996 Phys. Rev. Lett. 773443

[29] Duivenvoorden K and Quella T 2012 Phys. Rev. B 86235142

[30] Isakov S V, Hastings M B and Melko R G 2011 Nat. Phys. 7772

[31] Zhang Y, Grover T and Vishwanath A 2011 Phys. Rev. Lett. 107067202

[32] Li H and Haldane F D M 2008 Phys. Rev. Lett. 101010504

[33] Kitaev A Y 2003 Ann. Phys. 3032

[34] Takezoe H, Gorecka E and Čepič M A 2010 Rev. Mod. Phys. 82897

[35] Chubukov A V 1991 Phys. Rev. B 444693

[36] Heidrich-Meisner F, Honecker A and Vekua T 2006 Phys. Rev. B 74020403

[37] Smacchia P, Amico L, Facchi P, Fazio R, Florio G, Pascazio S and Vedral V 2011 Phys. Rev. A 84022304

[38] Montes S and Hamma A 2012 Phys. Rev. E 86021101

[39] Lee T E, Joglekar Y N and Richerme P 2016 Phys. Rev. A 94023610

[40] Jordan P and Wigner E 1928 Z. Phys. 47631

[41] Hill S and Wootters W K 1997 Phys. Rev. Lett. 785022

[42] Wootters W K 1998 Phys. Rev. Lett. 802245

[43] Lieb E, Shultz T and Mattis D 1961 Ann. Phys. 16407

[44] Barouch E, McCoy B M and Dresden M 1970 Phys. Rev. A 21075

[45] Barouch E and McCoy B M 1971 Phys. Rev. A 3786

[46] Wick G C 1950 Phys. Rev. 80268

[47] Pollmann F, Berg E, Turner A M and Oshikawa M 2012 Phys. Rev. B 85075125

[48] Osborne T J and Nielsen M A 2002 Phys. Rev. A 66032110

[49] Amico L, Osterloh A, Plastina F, Fazio R and Palma G M 2004 Phys. Rev. A 69022304

[50] Hiesmayr B C and Huber M 2008 Phys. Rev. A 78012342

[51] Ma Z-H, Chen Z-H, Chen J-L, Spengler C, Gabriel A and Huber M 2011 Phys. Rev. A 83062325

[52] Rafsanjani S M H, Huber M, Broadbent C J and Eberly J H 2012 Phys. Rev. A 86062303

[53] Nielsen M A and Chuang I 2001 Quantum Computation and Quantum Information (Cambridge: Cambridge University Press)

[54] Hastings M B and Wen X-G 2005 Phys. Rev. B 72045141

[55] Hamma A, Giampaolo S M and Illuminati F 2016 Phys. Rev. A 93012303

[56] Giampaolo S M and Zonzo G 2017 Phys. Rev. A 95012121

[57] Hartman T 2013 Entanglement entropy at large central charge (arXiv:1303.6955) 